\title{
Mathematical Assessment of the Dynamical Model of Smoking Tobacco Epidemic in Bangladesh
}

\author{
Sk. Abdus Samad ${ }^{\# 1}$, Md. Tusberul Islam² ${ }^{2}$, Sayed Toufiq Hossain Tomal ${ }^{3}$ and MHA Biswas ${ }^{4}$ \\ ${ }^{\# 1,4}$ Faculties, Mathematics Discipline, Khulna University, Khulna-9208, Bangladesh
}

\begin{abstract}
Bangladesh is one of the largest tobacco users in the world being troubled by smoking related issues. In this paper we consider a compartmental mathematical model of smoking in which the population is divided into five compartments: susceptible, expose, smokers, temporary quitters and permanent quitters described by ordinary differential equations. We study by including the conversion rate from light smoker to permanent quit smokers. The basic reproduction number $R_{0}$ has been derived and then we found two euilibria of the model one of them is smoking-free and other of them is smoking-present. We establish the positivity, boundedness of the solutions and perform stability analysis of the model. To decrease the smoking propensity in Bangladesh we perform numerical simulation for various estimations of parameters which offer understanding to give up smoking and how they influence the smoker and exposed class. This model gives us legitimate thought regarding the explanations for the spread of smoking in Bangladesh.
\end{abstract}

Keywords- Smoking, Compartments, Numerical Simulation, Tobacco and Secondary smoking.

\section{INTRODUCTION}

Tobacco consumption is a global public health concern. There are around 1 billion smokers on earth and it is expected to rise over 1.6 billion by 2025 [1]. Smoking is a practice in which a substance is burned and the resulting smoke breathed in to be tasted and absorbed into the bloodstream. Most commonly substance is the dried leaves of the tobacco plant which have been rolled into a small square of rice paper to create a small, round cylinder called a "cigarette" [28]. The World Health Organization estimate that each year tobacco cause about 6 million deaths(about $10 \%$ of all deaths) with 600,000 of these occurring in non smokers due to second hand smoke $[29,30]$. In the $20^{\text {th }}$ century tobacco is estimated to have caused 100 million deaths [30]. Cigarette smoke contains more than 7,000 chemicals. Inhaling cigarette smoke exposes the cigarette smoker to these numerous toxins, which include the various tobacco constituents and the products of paralysis $[2,3,18]$. Exposure to this complex chemical mixture causes immediate adverse physiologic effects shortly after the exposure occurs. The ultimate harm caused by exposure to the toxic agents in cigarette smoke is determined in large part by the extent of the exposure, and most adult cigarette smoker tend to smoke many cigarettes per day for decades $[2,4,5]$. This repeated inhalation of the complex mixture of cigarette smoke toxicants at high daily doses, often sustained over the course of many years, causes a broad spectrum of short-term and long-term health effects that affect most major organ systems [5, 6, 7]. In the short run, cigarette smoking causes the smoker to have overall diminished health status as measured by a diverse array of indices, including biomarkers of physiologic disadvantage, lower self-reported health, susceptibility to acute illnesses and respiratory symptoms and absence from school and work [5, 8]. Among the long-term health effects are smoking-caused diseases that are the major causes of death in middle- and upper-income nations: coronary heart disease, cancer, and chronic obstructive pulmonary disease, damages lung, reduces vision, oral diseases, weaken immune system, causes muscle deterioration, bone degeneration, and so on $[6,9]$.

Bangladesh is a lower middle income country with a densely population over 164 millions in a limited space. Smoking is very common in Bangladesh among all ages of people along with male and female. $35.3 \%$ of all adults (age 15+) use any tobacco product in which $46.0 \%$ are men and $25.2 \%$ are women $[10,31]$. Among young (ages 13-15), 6.9\% use tobacco in any form (boys 9.2\%, girls 2.8\%) and $4.5 \%$ use smokeless tobacco products (boys $5.9 \%$, girls $2.00 \%$ ) [11,31]. Second hand smoking is a severe problem in Bangladesh because $42.7 \%$ of adults who work indoors are exposed to secondhand smoke in the workplace, $49.7 \%$ of those who visit restaurants are exposed there and $44.0 \%$ of adults who use public transport are exposed while on it. $59.0 \%$ of youth (ages 13-15) are exposed to secondhand smoke in public places and $31.1 \%$ of youth are exposed to secondhand smoke at home $[12,29,31]$. Death rate due to smoking tobacco in Bangladesh is high for the older people $[19,21]$. This alarming situation causes over 113000 people die each year from tobacco using in 
Bangladesh around 23000 of these deaths are due to second hand smoking. Moreover there are about 1.2 million cases of tobacco-attributable illness $[13,31]$.

In Bangladesh there are so many causes' people to adopt smoking in all the sectors, ages and classes. Unemployment problem in Bangladesh causes frustration among young generation which leads them to adopt smoking whereas the upper class people take smoke as fashion and status. The cigarette is cheap and available everywhere both in cities and villages in Bangladesh. The lacking of entertainment in village pushes people to use tobacco and the young members of the family follow their elder and gradually addicted to smoking. [4, 14] The students in different educational institutions in Bangladesh addict to cigarette pursuing their friends and bad companies. In cities like Dhaka the percentage of smoking in slum dwellers is higher because of their lower living style and indifferent of health hazards. Alongside the male the female of Bangladesh are addicted to smoking at a moderate percentage. Among the garments workers and in the prostitutions the female are mostly addicted.

Long term tobacco uses create a great health hazards and it leads to various fatal diseases and illness among the general mass. For this Bangladesh has to face a serious economic loss every year that impacts greatly directly or indirectly in the national economy. The Global Adult Tobacco Survey (GATS) 2017 revealed the social gradient in tobacco use in Bangladesh in which prevalence increases with decreasing socioeconomic status: $24 \%$ of those in the highest wealth quintile use tobacco compared to $48 \%$ of those in the lowest wealth quintile, where households are more at risk of suffering catastrophic health expenditure and impoverishment through high healthcare costs from tobacco-related diseases, loss of productivity and opportunity costs [15]. The young generations are mostly seen to smoke tobacco. Day by day the young generation tends to smoke tobacco more and more and destructing their valuable lives and taking the country to the dark future.

In this paper we design and analyze a new general mathematical model of nonlinear differential equations to understand the smoking tobacco situation in Bangladesh based on [15] by adding another compartment of light smoker. The aim of this paper is quite specific, to study the designed model by using stability theory of the non linear differential equation with numerical simulations by using MATLAB solver ODE45. This paper is organized in the following manner: In section 1, introduction. In section two, formulation of mathematical model. In section 3, model analysis with euilibria and stability. In section 4, numerical result and discussion. In section 5, conclusion and finally we end the paper with relevant references.

\section{Formulation OF THE MODEL}

Mathematical modeling is the best way to understand the dynamical behavior of any system of nonlinear differential equations [23, 24]. In this paper a population size $N(t)$ is subdivided into five compartments: susceptible compartment $P(t)$ (individuals who are not smoking tobacco, but at a high risk of smoking tobacco), light smoking tobacco $E(t)$, smoker $S(t)$, temporary quite smoking $Q(t)$ and permanently quite smoking $R(t)$ (individuals who are fully determined not to smoke tobacco anymore). The probability rate of conversion from nonsmoker to smoker is $\beta$ and the probability of conversion from smoker to temporarily quit smoking is $\sigma$. We assume the natural birth rate $\mu_{0}$ different from the natural death rate $\mu$. The flow chart based on the above assumptions and the corresponding dynamical system is given by figure 1 .

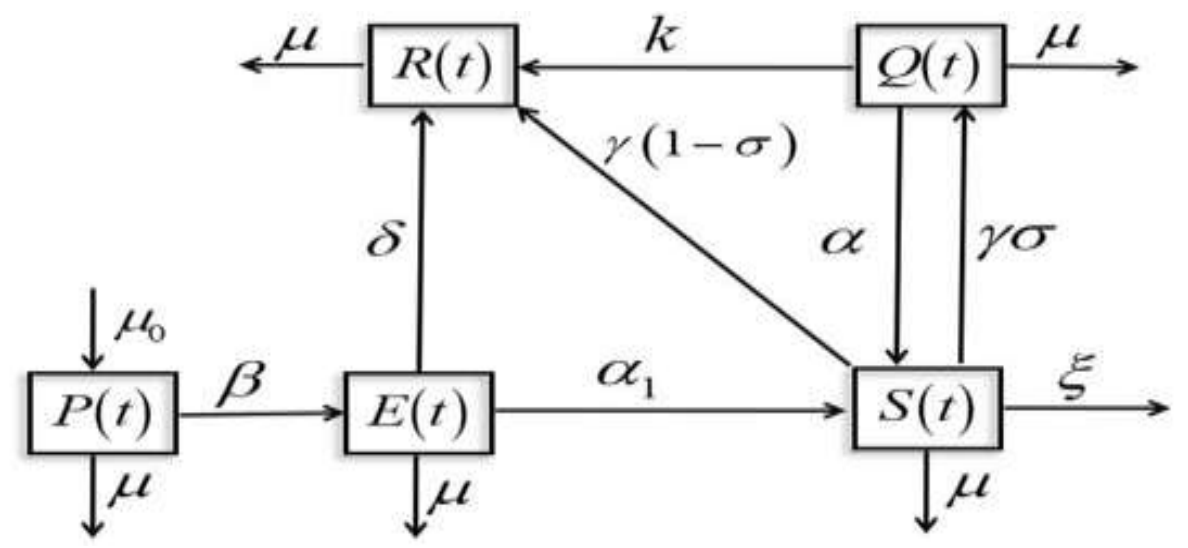

Figure 1: Compartmental model of smoking tobacco 
Based on the above diagram, the mathematical model can be represented by the following system of non-linear ordinary differential equations:

$$
\begin{aligned}
& \frac{d P(t)}{d t}=\mu_{0}-\mu P(t)-\beta E(t) P(t) \\
& \frac{d E(t)}{d t}=\beta P(t) S(t)-\left(\alpha_{1}+\delta+\mu\right) E(t) \\
& \frac{d S(t)}{d t}=\alpha_{1} E(t)+\alpha Q(t) S(t)-(\gamma+\mu+\xi) S(t) \\
& \frac{d Q(t)}{d t}=\gamma \sigma S(t)-(\alpha+\kappa+\mu) Q(t) \\
& \frac{d R(t)}{d t}=\kappa Q(t)+\gamma(1-\sigma) S(t)+\delta E(t)-\mu R(t)
\end{aligned}
$$

\section{MODEL ANALYSIS}

Model (1-5) has to be analyzed in order to describe the dynamics of smoking tobacco addiction. The objective of this analysis is to control the adverse situation from locality. For the analysis of model (1-5), a closed set has been considered

$$
\Omega=\left\{P(t), \mathrm{E}(t), \mathrm{S}(t), \mathrm{Q}(t), \mathrm{R}(t) \in \Re_{+}^{5} \mid 0 \leq N \leq \mu_{0} / \mu\right\}
$$

With the initial conditions, $P(0) \geq 0, E(0) \geq 0, S(0) \geq 0, Q(0) \geq 0, R(0) \geq 0$

\subsection{Boundedness and positivity of Solutions of the Model}

Two important concept of the solution of this model is boundedness and positivity. Now we have to show that the total population $N(t)=P(t)+E(t)+S(t)+Q(t)+R(t)$ is bounded for all $t \geq 0$. From models (1-5), the rate of change of total population is

$$
\begin{aligned}
& d N / d t=\mu_{0}-\mu(P(t)+E(t)+S(t)+Q(t)+R(t))-S(2 \gamma+\xi+\gamma \sigma) \\
& \Rightarrow d N / d t=\mu_{0}-\mu N-\mathrm{S} \xi \\
& \Rightarrow d N / d t+\mathrm{S} \xi=\mu_{0}-\mu N
\end{aligned}
$$

In the absence of smokers $(\mathrm{S}=0)$ so we get, $d N / d t \leq \mu_{0}-\mu N$. Solving this we have,

$$
N(t) \leq\left(\mu_{0} / \mu\right)-\mathrm{e}^{-\mu t}\left(\mu_{0}-N_{0} \mu\right) / \mu
$$

From this solution, it is clear that the total population $N(t)$ will approach the threshold $\mu_{0} / \mu$ as $t \rightarrow 0$.This implies that if the initial total population $N$ is less than $\mu_{0} / \mu$ if $N_{0} \leq \mu_{0} / \mu$. So definitely $\mu_{0} / \mu$ is the upper bound of $N$.

Theorem 1: All the solution of the differential equations (1-5) of the dynamical system is positive. Proof:

1. The solution of the equation (1) considering to the initial condition $P(0)=P_{0}, P(0)>0$ is

$$
P(t) \geq\left(\mu_{0}-\mathrm{e}^{-\mu t}\left(\mu_{0}-P_{0} \mu\right)\right) / \mu
$$

2. The solution of the equation (2) considering to the initial condition $E(0)=E_{0}, E(0)>0$ is

$$
E(\mathrm{t}) \geq E_{0} \mathrm{e}^{-t\left(\alpha_{1}+\delta+\mu\right)}
$$

3. The solution of the equation (3) considering to the initial condition $S(0)=S_{0}, S(0)>0$ is

$$
S(t) \geq S_{0} \mathrm{e}^{-t(\gamma+\mu+\xi)}
$$

4. The solution of the equation (4) considering to the initial condition $Q(0)=Q_{0}, Q(0)>0$ is

$$
Q(t) \geq Q_{0} \mathrm{e}^{-t(\alpha+\kappa+\mu)}
$$

The solution of the equation (5) considering to the initial condition $R(0)=R_{0}, R(0)>0$ is 
$R(t) \geq R_{0} \mathrm{e}^{-\mu t}$

Therefore, all the solutions (1-5) of the dynamic model of differential equations are positive.

\subsection{Smoking Free Equilibrium Point and Endemic Equilibrium Point}

Let us consider the smoking free equilibrium point is $\mathrm{w}_{0}$. In case of smoking free equilibrium point for the model, all the state variables $P(t), E(t), S(t), Q(t), R(t)$ are zero, except the susceptible compartments $\mathrm{S}$. By considering, $P(t)=E(t)=S(t)=Q(t)=R(t)=0$ we get, $\mu_{0}-\mu P=0, \Rightarrow P=\mu_{0} / \mu$. So, the smoking free equilibrium for the model is $w_{0}=(P, E, S, Q, R)=\left(\mu_{0} / \mu, 0,0,0,0\right)$.

Let the endemic equilibrium point for the model is $\mathrm{w}_{1}$ that can be obtained by considering $d P / d t=d E / d t=d S / d t=d Q / d t=d R / d t=0$ we get,

$\mu_{0}-\mu P-\beta E P=0, \beta P S-E\left(\alpha_{1}+\delta+\mu\right)=0, \alpha_{1} E+\alpha Q-S(\gamma+\mu+\xi)=0$,

$\gamma \sigma S-\alpha Q-(\kappa+\mu) Q=0, \delta E+\kappa Q-\mu R+\gamma(1-\sigma) S=0$

Then by solving, we get the endemic equilibrium point $P^{*}, E^{*}, S^{*}, Q^{*}, R^{*}$ are

$\left(P^{*}, E^{*}, S^{*}, Q^{*}, R^{*}\right)=\left(A_{1}, A_{2} p_{1} / \alpha \delta \sigma, \psi_{1} A_{1} / \gamma \sigma, A_{2}, A_{1}\left(\delta p_{1}+\kappa \alpha \delta \sigma+(1-\sigma) \psi_{1}\right) / \mu \alpha \delta \sigma\right)$

Where

$\psi_{1}=\alpha+\kappa+\pi, \psi_{2}=\gamma+\pi+\xi, \psi_{3}=\alpha_{1}+\delta+\mu, p_{1}=\psi_{1} \psi_{2}-\alpha \delta \sigma, A_{1}=\mu_{0} \alpha \delta \sigma /\left(\mu_{0} \alpha \delta \sigma+\beta p_{1} A_{2}\right)$

$A_{2}=\alpha \delta \sigma\left(\beta \mu_{0} \alpha \delta \sigma \psi_{1}-\mu \gamma \sigma p_{1} \psi_{3}\right)$

\subsection{Basic Reproduction Number}

The basic reproduction number $R_{0}$ is a threshold parameter for the stability of smoke free equilibrium [25]. If $R_{0}>1$, the number of smoker population will increase at a higher rate and for this smoking addiction appears for long time. Otherwise smoking will die out within shortest possible time [30]. For calculating the Basic Reproduction Number $R_{0}$ we've used Next Generation Matrix Method. So we're considering only the linearized ODEs in equation (2) and (3) having smoker population and then we calculated our transmitted and transited matrix denoted by $T$ and $V$ respectively.

$V^{-1}=\left(\begin{array}{cc}\frac{1}{\alpha_{1}+\delta+\mu} & 0 \\ \frac{\alpha_{1}}{\left(\alpha_{1}+\delta+\mu\right)(\gamma+\mu+\xi)} & \frac{1}{\gamma+\mu+\xi}\end{array}\right)$

Now, next generation matrix is given by,

$$
\begin{gathered}
T V^{-1}-\lambda I=\left(\begin{array}{cc}
\frac{\alpha_{1} \beta}{\left(\alpha_{1}+\delta+\mu\right)(\gamma+\mu+\xi)}-\lambda & \frac{\beta}{\gamma+\mu+\xi} \\
0 & -\lambda
\end{array}\right) \\
\text { So, }\left|T V^{-1}-\lambda I\right|=-\lambda\left(\frac{\alpha_{1} \beta}{\left(\alpha_{1}+\delta+\mu\right)(\gamma+\mu+\xi)}-\lambda\right)
\end{gathered}
$$

Find the characteristic equation and after solving we find the eigenvalues. The largest eigenvalue is the required basic reproduction number.

$$
R_{0}=\frac{\alpha_{1} \beta}{\left(\alpha_{1}+\delta+\mu\right)(\gamma+\mu+\xi)}
$$

\subsection{Stability Analysis}

Stability theory plays a central role in the field of dynamical system. The basic concept of stability emerged from the study of an equilibrium state of a system of differential equation [27]. 
We have to establish the local stability at drug free equilibrium point. To prove this, we need to use the following theorem.

Theorem 2: The smoke free equilibrium point is locally asymptotically stable if all the eigenvalues have negative algebraic sign and unstable otherwise.

Proof: In order to perform the stability analysis at drug free equilibrium point, we have from equations (1-5) $d P / d t=\mu_{0}-\mu P-\beta E P, d E / d t=\beta P S-E\left(\alpha_{1}+\delta+\mu\right), d S / d t=\alpha_{1} E+\alpha Q-S(\gamma+\mu+\xi) d Q / d t=\gamma \sigma S-\alpha Q$ $-(\kappa+\mu) Q, d R / d t=\delta E+\kappa Q-\mu R+\gamma(1-\sigma) S$

The Jacobian matrix of the system of ODEs is

$$
J=\left(\begin{array}{ccccc}
-\mu-\beta E & -\beta P & 0 & 0 & 0 \\
\beta S & -\alpha_{1}-\delta-\mu & \beta P & 0 & 0 \\
0 & \alpha_{1} & -\gamma-\mu-\xi & \alpha & 0 \\
0 & 0 & \gamma \sigma & -\alpha-\kappa-\mu & 0 \\
0 & \delta & \gamma(1-\sigma) & \kappa & -\mu
\end{array}\right)
$$

At the smoking free equilibrium point $w_{0}=\left(\mu_{0} / \mu, 0,0,0,0\right)$, the characteristic equation is

$$
\left|J\left(w_{0}\right)-\lambda I\right|=\left(\begin{array}{ccccc}
-\lambda-\mu & -\frac{\beta \mu_{0}}{\mu} & 0 & 0 & 0 \\
0 & -\alpha_{1}-\delta-\lambda-\mu & \frac{\beta \mu_{0}}{\mu} & 0 & 0 \\
0 & \alpha_{1} & -\gamma-\lambda-\mu-\xi & \alpha & 0 \\
0 & 0 & \gamma \sigma & -\alpha-\kappa-\mu-\lambda & 0 \\
0 & \delta & -\gamma(\sigma-1) & \kappa & -\lambda-\mu
\end{array}\right)
$$

$\left|J\left(w_{0}\right)-\lambda I\right|=C_{0} \lambda^{5}+C_{1} \lambda^{4}+C_{2} \lambda^{3}+C_{3} \lambda^{2}+C_{4} \lambda+C_{5}=0$ Where

$C_{0}=-1, C_{1}=-\left(\alpha-\alpha_{1}-\delta-\gamma-\kappa-\xi\right), C_{2}=-3 \alpha \mu-3 \alpha_{1} \mu-3 \delta \mu-3 \gamma \mu-4 \kappa \mu-3 \mu \xi-6 \mu^{2}-\alpha \alpha_{1}-\alpha \delta-\alpha \gamma-\alpha \alpha_{1}-\delta \gamma$ $-\alpha_{1} \kappa-\delta \kappa-\gamma \kappa-\alpha \xi-\alpha_{1} \xi-\delta \xi-\kappa \xi+\alpha \gamma \sigma+\left(\alpha_{1} \beta \mu_{0} / \mu\right), C_{3}=-\mu \tau-\alpha \phi-\delta(\gamma \kappa+\alpha \xi+\kappa \xi-\alpha \gamma \sigma+\alpha \gamma), C_{4}=-\mu^{2} \varepsilon$ $-2 \mu \theta+\alpha_{1} \beta \mu \mu_{0}+2 \alpha \alpha_{1} \beta \mu_{0}+2 \alpha_{1} \beta \kappa \mu_{0}, C_{5}=-\mu\left(\alpha \mu^{3}+\kappa \mu^{3}+\alpha \mu^{2} \xi+\kappa \mu^{2} \xi+\alpha \alpha_{1} \mu^{2}+\alpha \delta \mu^{2}+\alpha \gamma \mu^{2}+\alpha \kappa \mu^{2}+\delta \kappa \mu^{2}+\gamma\right.$ $\left.\kappa \mu^{2}+\alpha_{1} \gamma \kappa \mu+\delta \gamma \kappa \mu+\alpha \alpha_{1} \mu \xi+\alpha \delta \mu \xi+\alpha_{1} \kappa \mu \xi+\delta \kappa \mu \xi-\alpha \gamma \mu^{2} \sigma-\alpha \alpha_{1} \beta \mu_{0}+\alpha \alpha_{1} \gamma \mu+\alpha \delta \gamma \mu-\alpha_{1} \beta \kappa \mu_{0}-\alpha \alpha_{1} \gamma \mu \sigma-\alpha \delta \gamma \mu \sigma\right)$

According to the Routh-Hurwitz stability criterion if all the algebraic sign of the first column are same sign, this means all the roots have negative algebraic sign and this shows that the smoke free equilibrium point is stable.

Theorem 3: The endemic equilibrium point is locally asymptotically stable if $\lambda>1$.

Proof: We want to establish the stability analysis of equations (1-5) at endemic free equilibrium point. The Jacobian matrix is

$$
J=\left(\begin{array}{ccccc}
-\mu-\beta E & -\beta P & 0 & 0 & 0 \\
\beta S & -\alpha_{1}-\delta-\mu & \beta P & 0 & 0 \\
0 & \alpha_{1} & -\gamma-\mu-\xi & \alpha & 0 \\
0 & 0 & \gamma \sigma & -\alpha-\kappa-\mu & 0 \\
0 & \delta & \gamma(1-\sigma) & \kappa & -\mu
\end{array}\right)
$$

At endemic equilibrium point $w_{1}=\left(P^{*}, E^{*}, S^{*}, Q^{*}, R^{*}\right)$, we get characteristic equation 
$\left|J\left(w_{1}\right)-\lambda I\right|=\left|\begin{array}{ccccc}-\lambda-\mu-\beta e & -\beta p & 0 & 0 & 0 \\ \beta s & -\alpha_{1}-\delta-\mu-\lambda & \beta p & 0 & 0 \\ 0 & \alpha_{1} & -\gamma-\lambda-\mu-\xi & \alpha & 0 \\ 0 & 0 & \gamma \sigma & -\alpha-\kappa-\mu-\lambda & 0 \\ 0 & \delta & -\gamma(\sigma-1) & \kappa & -\lambda-\mu\end{array}\right|=0$

$\left|J\left(w_{0}\right)-\lambda I\right|=C_{0} \lambda^{5}+C_{1} \lambda^{4}+C_{2} \lambda^{3}+C_{3} \lambda^{2}+C_{4} \lambda+C_{5}=0$ where $C_{0}=1, C_{1}=-\alpha_{1}-\delta-\gamma-4 \mu-\xi-\beta e, C_{2}=\alpha \beta p-\delta \gamma$

$-2 \alpha_{1} \mu-2 \delta \mu-2 \gamma \mu-\alpha_{1} \xi-\delta \xi-2 \mu \xi-\mu\left(\alpha_{1}+\delta+\gamma+3 \mu+\xi+\beta e\right)-3 \mu^{2}-\beta^{2} p s-\alpha_{1} \beta e-\beta \delta e-\beta e \gamma-\alpha_{1} \gamma+\alpha_{1} \beta p$

$-2 \beta e \mu-\beta e \xi, C_{3}=\alpha \beta \mu p-\delta \mu^{2}-\gamma \mu^{2}-\mu^{2} \xi-\mu^{3}-\mu\left(\alpha_{1} \gamma+\delta \gamma+2 \alpha_{1} \mu+2 \delta \mu+2 \gamma \mu+\alpha_{1} \xi+\delta \xi+2 \mu \xi+3 \mu^{2}+\right.$ $\left.\beta^{2} p s+\alpha_{1} \beta e+\beta \delta e+\beta e \gamma-\alpha \beta p-\alpha_{1} \beta p+2 \beta e \mu+\beta e \xi\right)-\alpha_{1} \gamma \mu-\delta \gamma \mu-\alpha_{1} \mu \xi-\delta \mu \xi-\beta e \mu^{2}-\alpha_{1} \beta e \xi-\alpha_{1} \mu^{2}$ $+\alpha_{1} \beta \mu p-\beta \delta e \xi-\beta e \mu \xi+\alpha \beta^{2} e p+\alpha_{1} \beta^{2} e p-\beta^{2} \gamma p s-\beta^{2} \mu p s-\beta^{2} p s \xi-\alpha_{1} \beta e \gamma-\beta \delta e \gamma-\alpha_{1} \beta e \mu-\beta \delta e \mu-\beta e$ $\gamma \mu, C_{4}=-\mu\left(\alpha_{1} \mu^{2}+\delta \mu^{2}+\gamma \mu^{2}+\mu^{2} \xi+\mu^{3}+\alpha_{1} \gamma \mu+\delta \gamma \mu+\alpha_{1} \mu \xi+\delta \mu \xi+\beta e \mu^{2}+\alpha_{1} \beta e \xi-\alpha \beta \mu p-\alpha \alpha_{1} \beta \mu p+\beta \delta e \xi\right.$ $\left.+\beta e \mu \xi-\alpha \beta^{2} e p-\alpha_{1} \beta^{2} e p+\beta^{2} \gamma p s+\beta^{2} \mu p s+\beta^{2} p s \xi+\alpha_{1} \beta e \gamma+\beta \delta e \gamma+\alpha_{1} \beta e \mu+\beta \delta e \mu+\beta e \gamma \mu\right)$

Applying the Routh-Hurwitz stability criterion, we get

$\lambda^{3} \lambda^{4} \mid \begin{array}{ccc}C_{0} & C_{2} & C_{4} \\ C_{1} & C_{3} & 0 \\ -\frac{C_{0} C_{3}-C_{3} C_{2}}{C_{1}} & C_{4} & 0 \\ \frac{C_{1}\left(C_{1} C_{4}^{\prime}+\frac{C_{3}\left(C_{0}^{\prime} C_{3}-C_{1} C_{2}\right)}{C_{1}}\right)}{C_{0}^{\prime} C_{3}-C_{1} C_{2}} & 0 & 0\end{array}$

$\left(C_{4} C_{1}^{2}-C_{2} C_{1} C_{3}+C_{0} C_{3}^{2}\right) /\left(C_{0} C_{3}-C_{1} C_{2}\right)>0, \Rightarrow C_{4} C_{1}^{2}-C_{2} C_{1} C_{3}+C_{0} C_{3}^{2}>0, \Rightarrow C_{4} C_{1}^{2}+C_{0} C_{3}^{2}>C_{2} C_{1} C_{3}$

All the roots have negative real parts for the condition $C_{4} C_{1}^{2}+C_{0} C_{3}^{2}>C_{2} C_{1} C_{3}$. Hence the endemic equilibrium point is stable.

\section{NUMERICAL RESULTS}

We have solved the model numerically based on the parameters present in the system of equations (15). The simulations are carried out using MATLAB programming language by the set of parameter values shown in Table 1. This simulation is to explore the behaviors of our model and to describe the dynamics of smoking tobacco.

\begin{tabular}{|l|l|l|l|}
\hline No & Parameter & Values & Description \\
\hline 1 & $\beta$ & 0.6534 & Rate of conversion from non-smoker to smoker [estimated] \\
\hline 2 & $\gamma$ & 0.4 & $\begin{array}{l}\text { Rate of return from smoker to temporarily quit and permanent quit smokers } \\
\text { [estimated] }\end{array}$ \\
\hline 3 & $\alpha$ & $0.1-0.4$ & Rate of return back from temporarily quit smoking to smoker [estimated] \\
\hline 4 & $\kappa$ & 0.01 & $\begin{array}{l}\text { Rate of conversion from temporarily quit smoking to permanent quit smokers } \\
\text { [estimated] }\end{array}$ \\
\hline 5 & $\alpha$ & $0.15-0.45$ & \begin{tabular}{l} 
The rate of conversion from exposed to smoker class [estimated] \\
\hline 6
\end{tabular} \\
\hline 7 & $\mu_{0}$ & $0.005-0.02$ & The rate of conversion from exposed to permanent quit smoker [assumption] \\
\hline
\end{tabular}




\begin{tabular}{|l|l|l|l|}
\hline 8 & $\mu$ & 0.02 & Natural death rate [32] \\
\hline 9 & $\xi$ & 0.015 & Smoking cause death rate[31] \\
\hline 10 & $\sigma$ & $0.6-0.9$ & The probability of conversion from smoker to temporarily quit smoking [estimated] \\
\hline
\end{tabular}

Table 1: This Table shows the value of parameters

Model has been solved numerically considering 10 years time period using the parameters from Table 1. We have simulated the effects on smoking for the parameters $\alpha_{1}$ (the rate of conversion from exposed to smoker class), $\delta$ (the rate of conversion from exposed to permanent quit smoker), $\alpha$ (rate of return back from temporarily quit smoking to smoker), $\sigma$ (the probability of conversion from smoker to temporarily quit smoking). The recovered and permanently quit smoking populations are affected by the parameters where smoking exists. We have simulated this parameters because the rate of conversion from exposed to smoker class, rate of return back from temporarily quit smoking to smoker and the probability of conversion from smoker to temporarily quit smoking enhance smoking habit. The rate of conversion from exposed to permanent quit smoker also has a slight impact on prevalence of smoking. If those rates and probability can be minimized then the smoker class will must decrease. The above table 1 represented the value of parameters used in all our simulations. We change the value of $\alpha_{1}$ (the rate of conversion from exposed to smoker class) and keeping the other parameter fixed. The simulations results in this case are shown in Figure 2.

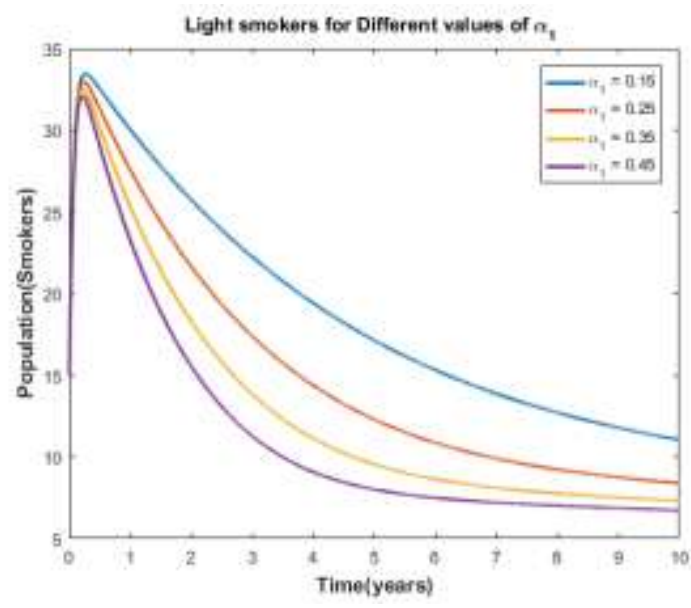

Figure 2: Variation of Light smoker population for different values of $\alpha_{1}$.

The Figure 2 is going from the exposed towards permanent smokers. We can plainly observe the bend are getting lower with the expansion of $\alpha_{1}$. So we can compose that the number of inhabitants in exposed class is diminishing by the expanding of the parameter keeping different parameters fixed. So also, we can say that the light smoker populace is expanding when diminishing.

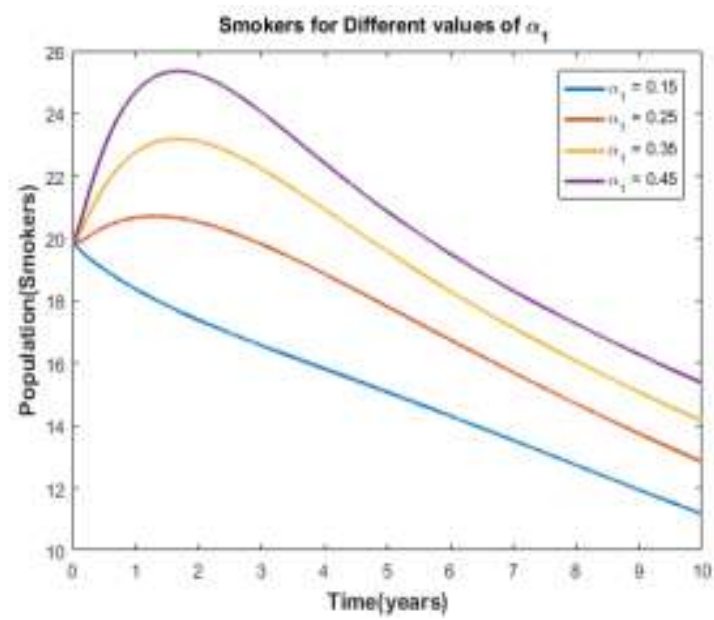

Figure 3: Variation of permanent smoker population for the different values of $\alpha_{1}$.

In Figure 3 the variation of regular smoker has been represented for different values of $\alpha_{1}$ keeping other parameter value fixed. It can be revealed that when $\alpha_{1}$ (the rate of conversion from exposed to permanent smokers) decrease, we can see that the number of smoker decrease immensely. 


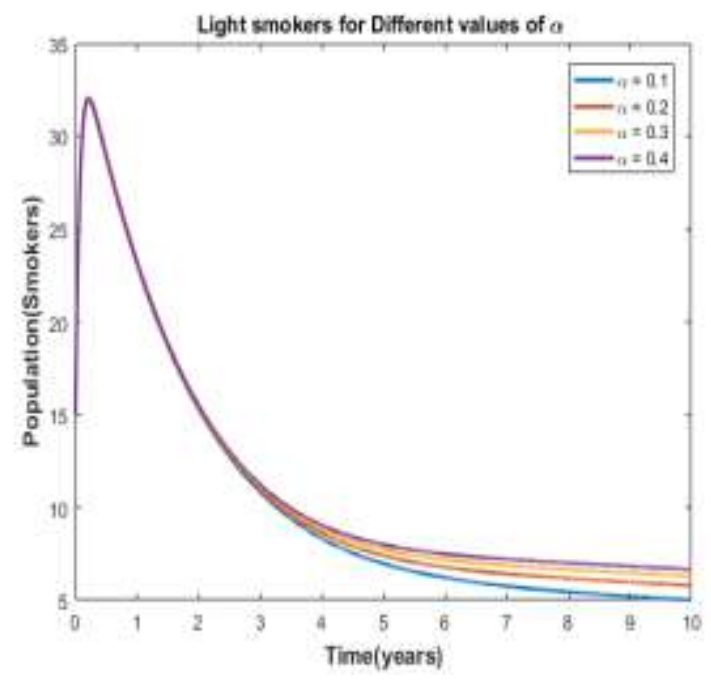

Figure 4: Variation of Light smoker population for the different values of $\alpha$.

In Figure 4, we can see that, light smoker population is increasing with the increase of $\alpha$ (the rate of return back from temporarily quit smoking to smoker class. That means the smokers gave up smoking, if they start smoking again then number of smoker will increase.

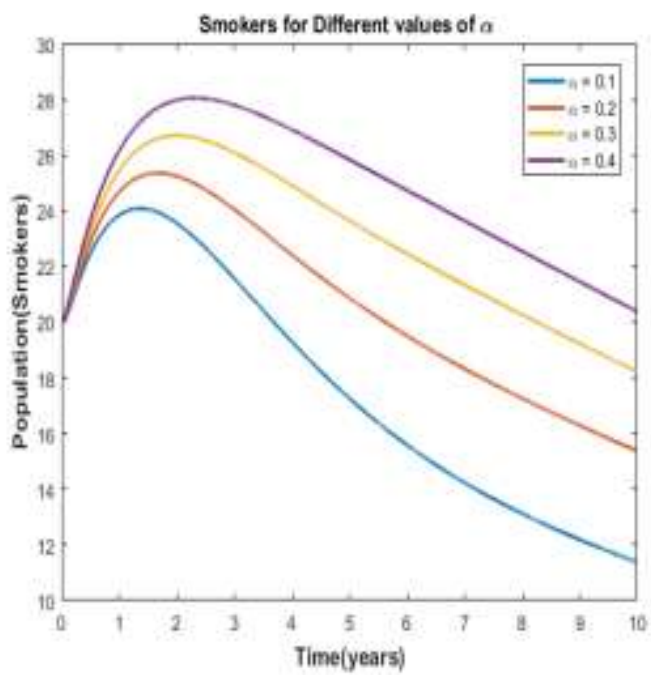

Figure 5: Variation of smoker population for the different values of $\alpha$.

From the Figure 5 for the increment of $\alpha$ (rate of return back from temporarily quit smoking to smoker) the smoker population is increasing. If the population gave up smoking previously and again start smoking permanently then the smoker population $S$ will increase more than previous.

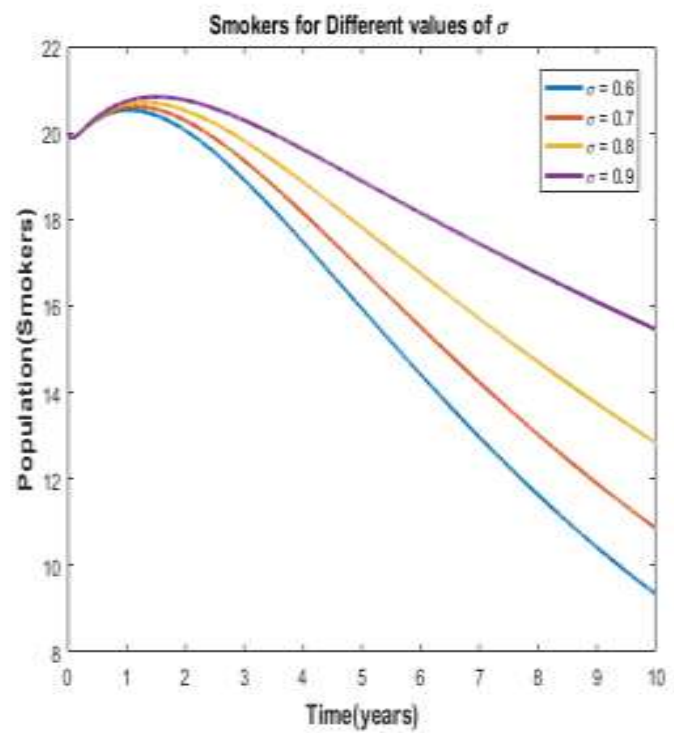

Figure 7: Variation of smoker population for the different values of $\sigma$.

(recovered) for the parameter $\alpha$.

Figure 6 shows that the recovered will increase for the increment of the rate of return back from temporarily quit smoking to smoker. The probability of conversion from smoker to temporarily quit smoking $\sigma$ has impact on smoker population. 


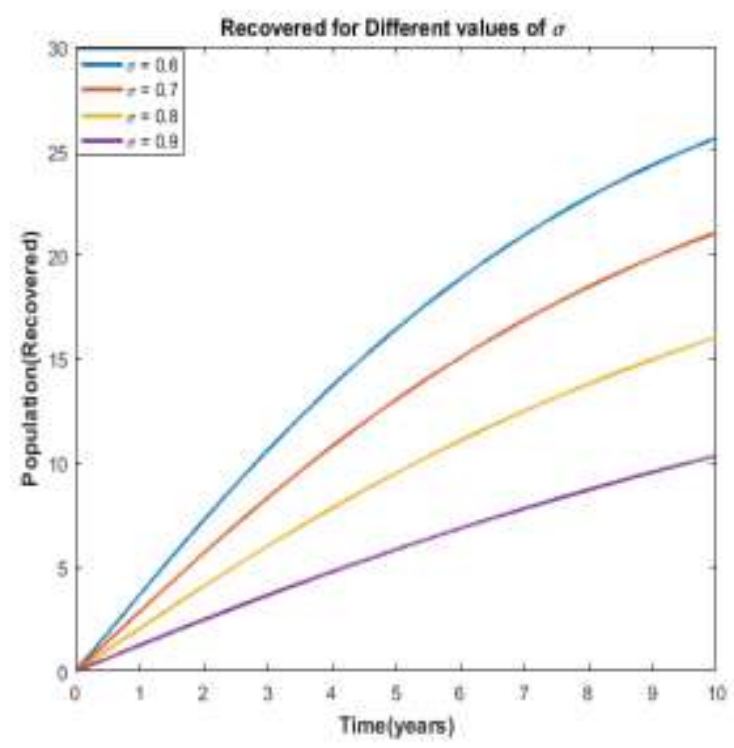

Figure 8: Variation of recovered population for the different values of $\sigma$.

From the variation of the probability of conversion from smoker to temporarily quit smoking in Figure 8 we have found that the increasing of the probability of conversion from smoker to temporarily quit smoking causes in the decrease of the recovered population. Similarly the decrease of $\sigma$ increases recovered population.

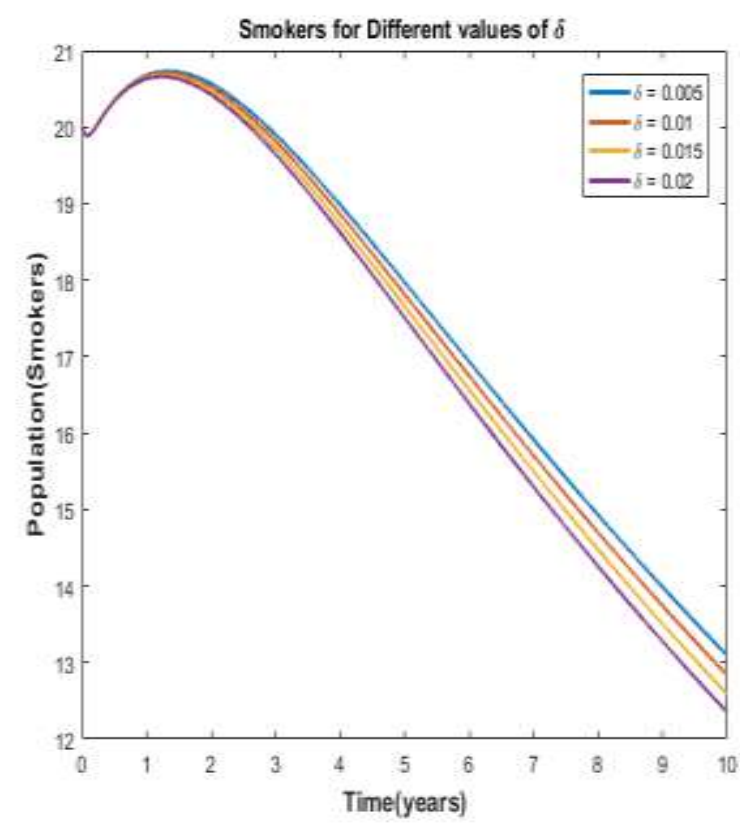

Figure 10: Variation of smoker population for the different values of $\delta$.

Figure 10 shows the smokers decreasing dynamical behavior for the increment of $\delta$ the rate of conversion from exposed to permanent quit.

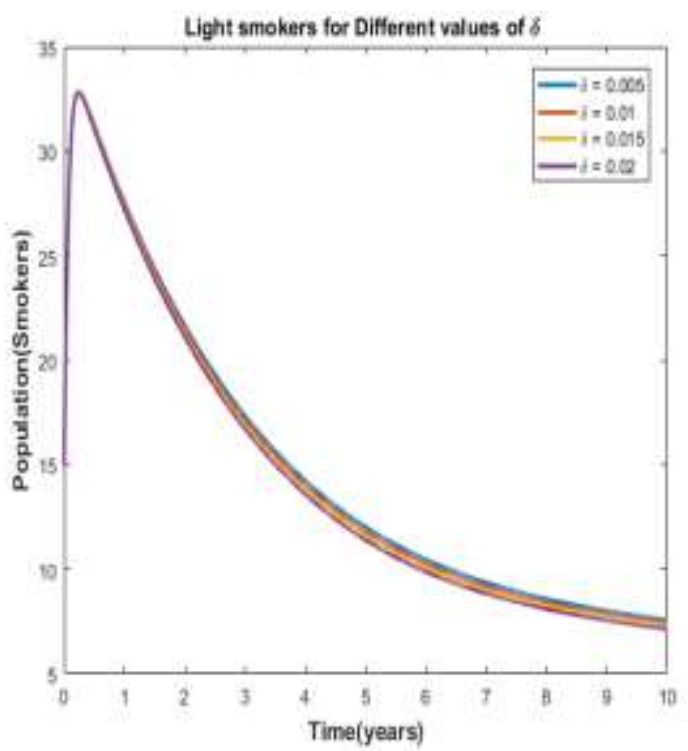

Figure 9: Variation of Light smoker population for the different values of $\delta$.

Here from the Figure 9 we can see, for the variation of the rate of conversion from exposed to permanent quit smoker makes effect on the exposed group. The increment of the rate of conversion from exposed to permanent quit smoker decrease exposed population when we keep the other parameters unchanged.

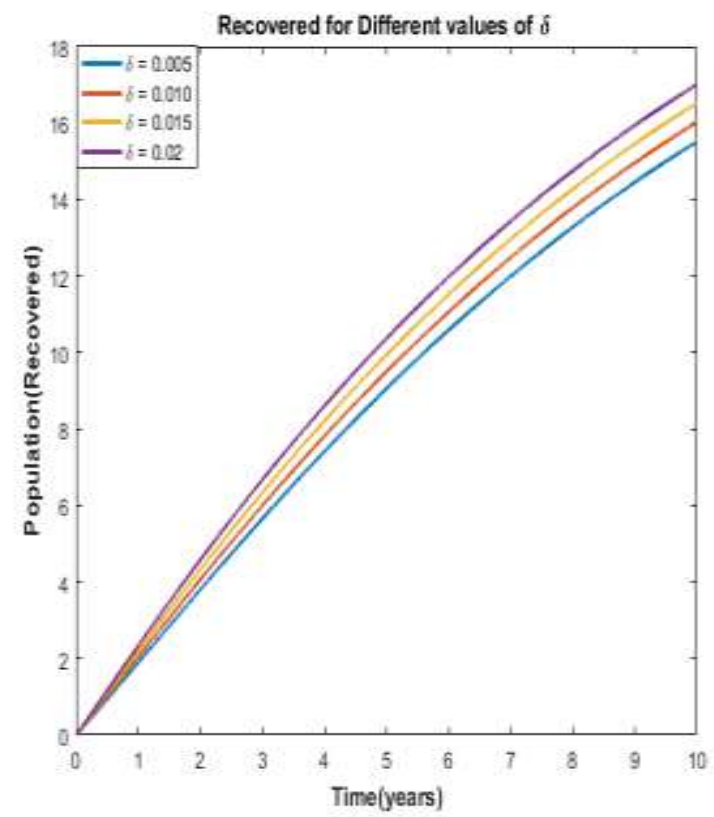

Figure 11: Variation of recovered population for the different values of $\delta$.

From the above Figure 11 we can see that the increment of recovered population changes with the increase of $\delta$ (the rate of conversion from exposed to permanent quit).

Page 44 


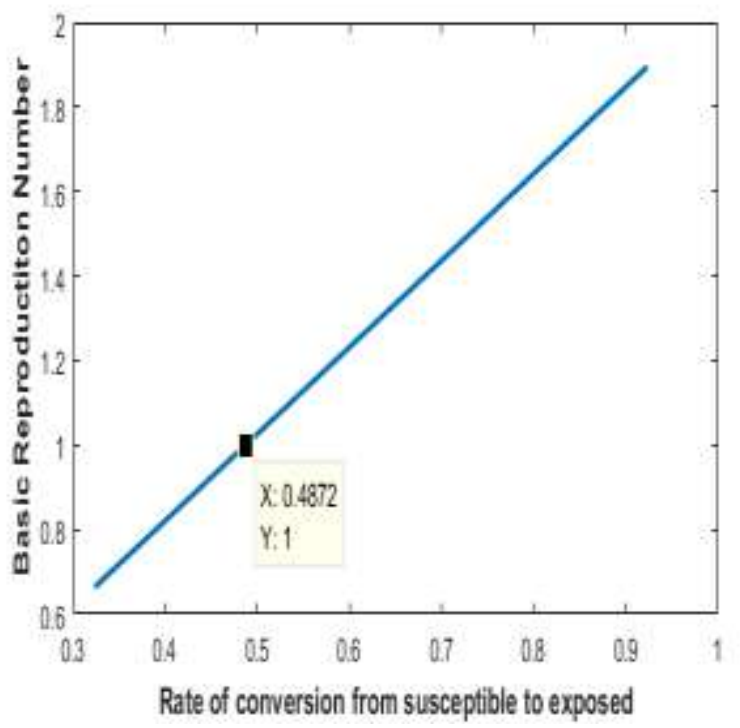

Figure 12: Rate of transmission from susceptible to exposed

From this graph we can see that when transmission rate $\beta=0.4872$ then the reproduction number $R_{0}=1$. This means that the smoking habit spreads in the society when $\beta$ exists 0.4872 .

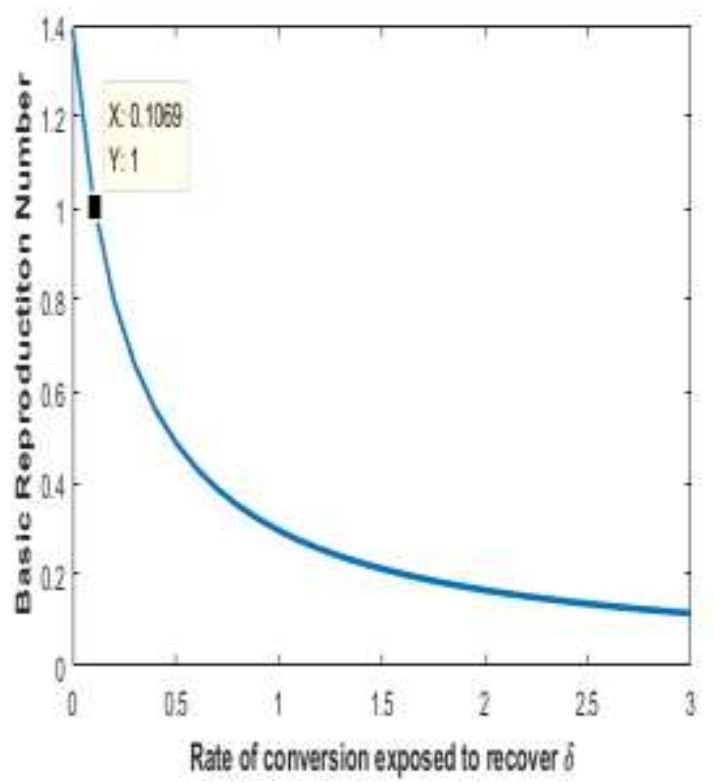

Figure 14: Rate of transmission from Exposed to recovered

From this graph we can see that when transmission rate $\delta>0.1069$ then the reproduction number $R_{0}>1$. This means that the smoking habit spreads in the society when $\delta$ exists 0.1069 .

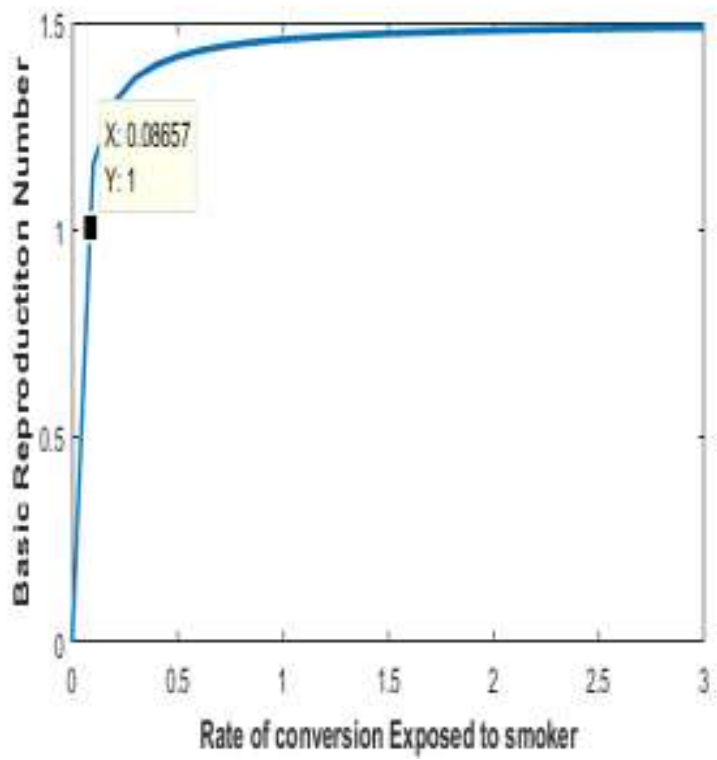

Figure 13: Rate of transmission from Exposed to smoker

From this graph we can see that when transmission rate $\alpha_{1}>0.08657$ then the reproduction number $R_{0}>1$. This means that the smoking habit spreads in the society when $\beta$ exists 0.08657 .

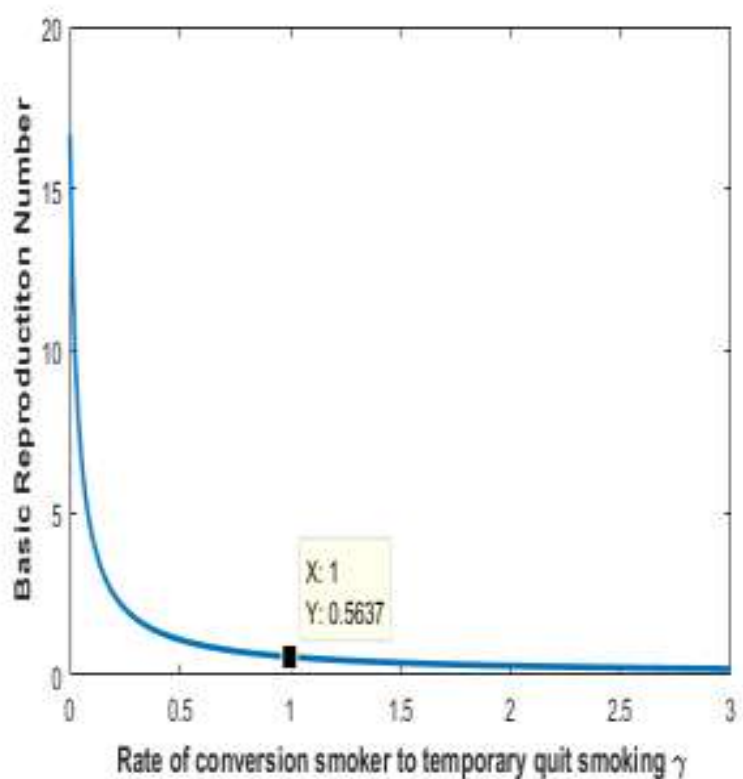

Figure 15: Rate of transmission from smoker to temporary quit smoking

From this graph we can see that when transmission rate from smoker to temporary quit smoking $\Upsilon>0.5637$ then the reproduction number $R_{0}>1$. This means that the smoking habit spreads in the society when $\Upsilon$ exists 0.5637 . 


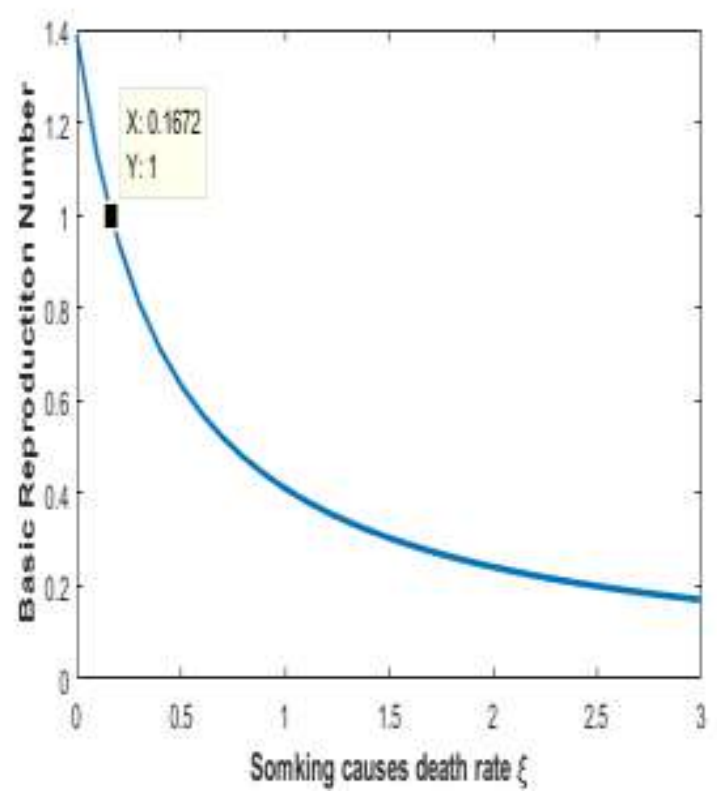

Figure16: Rate of transmission for smoking causes death rate

From this graph we can see that when transmission rate for smoking causes death rate $>0.1672$ then the reproduction number $R_{0}>1$. This means that the smoking habit spreads in the society when the smoking cause death rate exists 0.1672 .

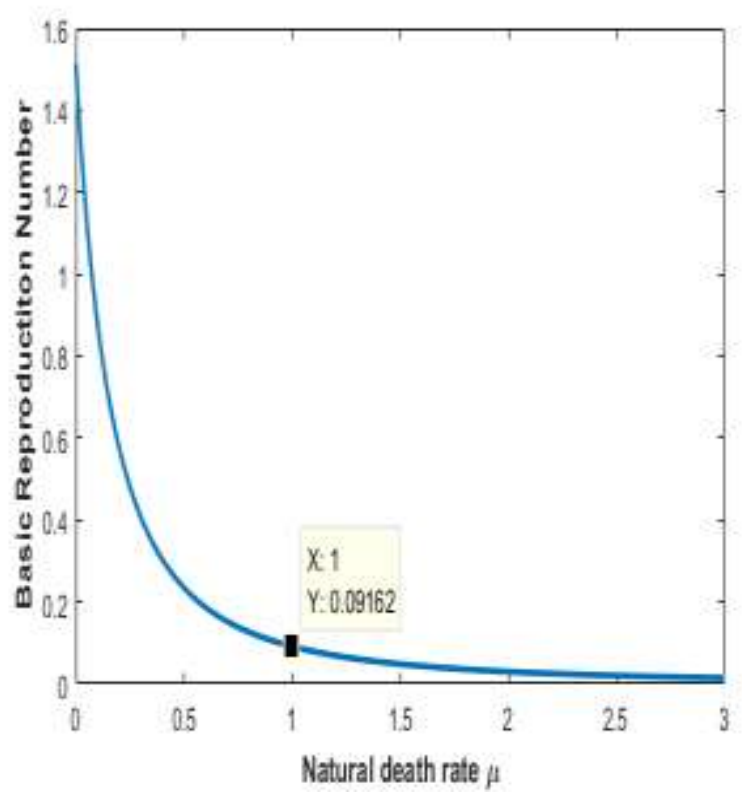

Figure 17: Rate of transmission for natural death rate

From this graph we can see that when $\mu_{0}>0.09162$ then the reproduction number $R_{0}>1$. This means that the smoking habit spreads in the society when the natural death rate exists 0.09162 .

\section{DISCUSSION AND CONCLUSION}

In this work, we present the behavior of smoking tobacco epidemic model, focused on the parameters that are mainly responsible for spreading smoking tobacco in our society. We have considered systems of nonlinear differential equations to study the dynamics of smoking. Extending a compartmental mathematical model on dynamics of smoking tobacco we found the generators of this model that gives us proper idea about the reasons behind the spread of smoking in our society. If the basic reproduction number is greater than unity then smoking spreads in our society. On the other hand if the basic reproduction number is less than unity then the smoking habit is low in the community. We have used next generation matrix method to determine the basic reproduction number of our extended model, $R_{0}=\alpha_{1} \beta /\left(\alpha_{1}+\delta+\mu\right)(\gamma+\mu+\xi)$ depending on our parameters. From the numerical analysis we can found that when the rate of conversion from susceptible to exposed $\beta>0.4872$ then the basic reproduction number $R_{0}>1$. That means smoking habit in our society is increasing when $\beta$ exceeds 0.4872 . The basic reproduction number $R_{0}=1$ when the conversion rate $\alpha_{1}=0.08657$. That means the smoking habit spread in our society when the rate of conversion from exposed to smoker class exceeds 0.08657 . When the rate of conversion from exposed to recovered $\delta>0.1069$ then the basic reproduction number $R_{0}>1$. That means smoking habit in our society is increasing when $\delta$ exceeds 0.1069 . When the rate of conversion from smoker to temporary quit smoking $\Upsilon>0.5637$ then the basic reproduction number $R_{0}>1$. That means smoking habit in our society is increasing when $\Upsilon$ exceeds 0.5637 . When transmission rate for smoking causes death rate $\xi>0.1672$ then the basic reproduction number $R_{0}>1$. That means smoking habit in our society is increasing when $\xi$ exceeds0.1672. When $\mu>0.09162$ then the basic reproduction number $R_{0}>1$. That means smoking habit in our society is increasing when $\mu$ exceeds 0.09162 .

In the model, the population of exposed (light smokers) class is decreasing by the increasing of the parameter $\alpha_{1}$ (the rate of conversion from exposed to smoker class). When $\alpha_{1}$ (the rate of conversion from exposed to permanent smokers) decrease, we can see that the number of smoker decrease immensely. The light smoker population is increasing with the increase of $\alpha$ (the rate of return back from temporarily quit smoking to smoker class). Also for the increment of $\alpha$ (rate of return back from temporarily quit smoking to smoker) the 
smoker population is increasing. $\sigma$ (Probability of conversion from smoker to temporarily quit smoking) increases then at the end smoker population will increase. Again for the increasing of the probability of conversion from smoker to temporarily quit smoking $\sigma$ causes in the decrease of the recovered population. The increment of the rate of conversion from exposed to permanent quit smoker $\delta$ decrease exposed population. Also the smokers decrease for the increment of $\delta$. We can see for the increment of $\delta$ (the rate of conversion from exposed to permanent quit) recovered population also increase.

The model strongly indicates that the spread of smoking tobacco depends on the transmission rate of parameters. To decrease the number of smokers in our society we must monitor the parameter so that $\beta$ not to exceed $0.4872, \alpha_{1}$ not to exceed $0.08657, \gamma$ not less than $0.5637, \delta$ not less than 0.1069 , $\xi$ not to less than 0.1672.From our perception the primary components of smoking tobacco are transformation between individuals of exposed and the smokers. So we should be quit Smoking by guardians around home and smoking around open assistance zone. Caution individuals about the threats of smoking tobacco, government ought to authorize the law for tobacco publicizing, sponsorship. Government ought to uphold the tobacco control approach to control smoking in open zone.

\section{REFERENCES}

[1] M. M. H. Khan, A. Khan, A. Kraemer, and M. Mori, "Prevalence and correlates of smoking among urban adult men in Bangladesh: slum versus non-slum comparison”, BMC Public Health, 9:149, doi: 10.1186/1471-2458-9-149, 2009.

[2] Z. Alkhudhari, S. Al-Sheikh, and S. Al-Tuwairqi, "The effect of occasional smokers on the dynamics of a smoking model", International Mathematical Forum, 9(25): 1207-1222, 2014.

[3] M. A. Kabir, K. L. Gohand, and M. M. H. Khan, "Tobacco Consumption and Illegal Drug Use among Bangladeshi Males: Association and Determinants", American Journal of Men's Health, 7(2): 128-137, 2012

[4] M. M. H. Khan, K. Aklimunnessa, M. A. Kabir, M. Kabir and Mitsuru Mori, "Tobacco consumption and its association with illicit drug use among men in Bangladesh”, Society for the study of addiction, 101: 1178-1186, 2006.

[5] R. J. Bonnie, K. Stratton, and L. Y. Kwan, "Public Health Implications of Raising the Minimum Age of Legal Access to Tobacco Products", National Academies Press (US), July 23, 2015.

[6] Z. Alkhudhari, S. Al-Sheikh, and S. Al-Tuwairqi, "The effect of heavy smokers on the dynamics of a smoking model", International Journal of Differential Equations and Applications, 14(4): 1311-2872, 2015.

[7] T. Rastogi, p. Jha, K. Reddy, D. Prabhakaran, D. Spiegelman, M. Stampfer, W. Willett, and A Ascherio, "Bidi and cigarette smoking and risk of acute myocardial infraction among males in urban India", Tobacco Control, 14: 356-358, 2005.

[8] G. Zaman, "Qualitative Behavior of Giving up Smoking Model”, Bulletin of the Malaysian Mathematical sciences society, 34: 403$415,2011$.

[9] D. Efroymson, S. Ahmed, J. Townsend, S. M. Alam, A. R. Dey, R. Saha, B. Dhar, A. I. Sujon, K. U. Ahmed, and O. Rahman, "Hungry for tobacco: an analysis of the economic impact of tobacco consumption on the poor in Bangladesh", Tobacco control, 10: 212-217, 2001.

[10] M. J. Husain, M. V. Baker, M. Parascandola, B. H. Khondker and I. B. Ahluwalia, "Money Gone Up in Smoke: The Tobacco Use and Malnutrition Nexus in Bangladesh”, Ann Glob Health, 82(5): 749-759, 2016.

[11] J. Bush, M. White, J. Kai, J. Rankin, and R. Bhopal, "Understanding influences on smoking in Bangladeshi and Pakistani adults: community based, qualitative study”, BMJ, 326 (7396): 962, DOI: 10.1136/bmj.326.7396.962, 2005.

[12] N. Nargis, M. E. Thompson, G. T. Fong, P. Driezen, A. K. M. G. Hussain, U. H. Ruthbah, A. C. K. Quah, and A. S. Abdullah, "Prevalence and Patterns of Tobacco Use in Bangladesh from 2009 to 2012: Evidence from International Tobacco Control (ITC) Study”, PLOS ONE, DOI:10.1371/journal.pone.0141135, 2015.

[13] M. S. Rahman, M. N. I. Mondal, M. R. Islam, M. M. Rahman, M N. Hoque and M. S. Alam, "Determinant factors of tobacco use among ever-married men in Bangladesh", Drug, Healthcare and Patient Safety, 2015(7): 77-85, 2015.

[14] M. S. Flora, C. G. N. Mascie-Taylor and M. Rahman, "Gender and locality differences in tobacco prevalence among adult Bangladeshis", Tobacco Control, 18:445-450, 2009.

[15] O. Sharomi, and A. B. Gumel, "Curtailing smoking dynamics: A mathematical modeling approach", Applied Mathematics and Computation, Elsevier, 195: 475-499, 2008.

[16] M. A. Adhana, and T. T. Mekonnen, "A Mathematical Model Analysis of Smoking Tobacco in the Case of Haremaya Town; Ethiopia", International Journal of Research Studies in Science, Engineering and Technology, 6(2): 14-24, 2019. 
[17] M. H. A. Biswas, T. Rahman and N. Haque, "Modeling the Potential Impacts of Global Climate Change in Bangladesh: an Optimal Control Approach", Journal of Fundamental and Applied Sciences, 8(1): 1-19, 2016.

[18] Z. Alkhudhari, S. Al-Sheikh, and S. Al-Tuwairqi, "The Effect of Heavy Smokers on the Dynamics of a Smoking Model", International Journal of Differential Equations and Applications, 14(4): 343-356, 2015.

[19] D. S. Alam, P. Jha, C. Ramasundarahettige, P. K. Streatfield, L. W. Niessen, M. A. H Chowdhury, A. T. Siddiquee, S. Ahmeda and T. G. Evans, "Smoking-attributable mortality in Bangladesh: proportional mortality study", Bull World Health Organ, 91(10):757-764, 2013.

[20] J. Ali, M. Faheem, A. M. Gul, Shahzeb, S. F. Abass and M. Hafizullah, "Frequency of Tobbaco use in Different Occupational Groups of Peshawar", International Journal of Pathology, 10(1): 9-12, 2012.

[21] World Health Organization report on the global tobacco epidemic: country profile Bangladesh, 2019.

[22] A. Zeb, G. Zaman, and S. Momani, "Suqare-root dynamics of a giving up smoking model, Applied Mathematical Modeling, 37(7): 5326-5334, 2013

[23] S. A. Samad, and M. H. A. Biswas, "Mathematical Understanding of Dynamical model for the Transmission of Endemic Tuberculosis in Bangladesh", Advanced Modeling and Optimization, 20 (2): 329-344, 2018.

[24] M. H. A. Biswas, L. T. Paiva, and M. D. R. De Pinho, "A SEIR Model for Control of Infectious Disease with Constraints", Mathematical bioscience and engineering, 11 (4): 761-784, 2014.

[25] M. H. A. Biswas, "Model and Control Strategy of the Deadly Nipah Virus (NiV) Infections in Bangladesh", Research and Review in Bioscience, 6 (12): 370-377, 2012.

[26] P. V. D. Driessche, and J. Watmough, "Reproduction numbers and sub-threshold endemic euilibria for compartmental models of disease transmission", Mathematical Biosciences, 180: 29-48, 2002.

[27] G. Chen, "Stability of Nonlinear System", Encyclopedia of RF and Microwave Engineering, Research Gate, DOI: 10.1002/0471654507.eme413, 2005.

[28] Wikipedia (2019), Passive Smoking [online], Available: https://en.wikipedia.org/wiki/Passive_smoking

[29] World Health Organization (2019), Tobacco Control in Bbangladesh [online], Available: http://www.searo.who.int/bangladesh/tobacco-control/en/

[30] Wikipedia (2019), Smoking [online], Available: https://en.wikipedia.org/wiki/Smoking

[31] The toll of tobacco in Bangladesh [online], Available: https://www.tobaccofreekids.org/problem/toll-global/asia/bangladesh

[32] Bangladesh Bureau of Statistics (BBS) [Online], Available: http://www.bbs.gov.bd/

[33] U. Bommisetty and M. Rajamane, "Impact of Recent Advances in Ergonomic Design and Digital Human Models in Industrial Environment”, International Journal of Science and Management Studies (IJSMS), 1(2): 14-19, 2018.

[34] J. P. Cabalo and M. M. Cabalo, "Mathematical Competencies and Character Traits Teachers in Relation to Pupils Academic Performance", International Journal of Science and Management Studies (IJSMS), 2(2): 139-154, 2019. 